\title{
Teaching Participatory Action Research: Fostering Impact ${ }^{1}$
}

\author{
Matthew Thomas Johnson (ORCID: 0000-0002-9987-7050; m.johnson@lancaster.ac.uk), ${ }^{2}$ \\ Victoria Gallagher ${ }^{3}$ and Roger Appleton ${ }^{4}$
}

This is an Author's Original Manuscript (AOM) of an article published by Taylor \& Francis in Political Science Teaching on 06 June 2019, available online: http://dx.doi.org/10.1080/15512169.2019.1616551

\begin{abstract}
Participatory action research (PAR) investigates issues through collaboration and cooperation between academics and non-academic community members. Recently, awareness of such approaches has increased as the need for transformative research and contribution to social goods has become clear. However, participatory methods have been deployed unevenly within the Humanities and Social Sciences and opportunities for engagement have been explored unevenly. This article seeks to assist colleagues in teaching PAR methods by reflecting upon our practice of working with Early Career Researchers (ECRs) to identify, explore and deploy participatory methods in ways that foster genuine social change and meaningful research trajectories.

Keywords: Participatory Action Research; Postgraduate Research Training; Early Career Researchers; Research Methods; Transformative research
\end{abstract}

The concept of participatory action research (PAR) has gradually gained traction as a viable approach in the Social Sciences and Humanities. In its most concrete form, PAR

involves researchers and participants working together to understand a problematic situation and change it for the better. There are many definitions of the approach, which share some common elements. PAR focuses on social change that promotes democracy and challenges inequality; is context-specific, often targeted on the needs of a particular group; is an iterative cycle of research, action and reflection; and often seeks to 'liberate' participants to have a greater awareness of their situation in order to take

\footnotetext{
${ }^{1}$ Ethical Approval was provided by Lancaster University Faculty of Arts and Social Sciences and Lancaster University Management School Research Ethics Committee (Reference number: FL17018).

${ }^{2}$ Matthew Johnson is Senior Lecturer in Politics at Lancaster University. His research focuses on the relationship between culture, public policy and wellbeing in issues such as austerity, Brexit and national rebuilding. He led the international, interdisciplinary, participatory study entitled 'A Cross-cultural Working Group on "Good Culture" and Precariousness', which involved embedded exchanges between community members from Ashington, Northumberland and Aboriginal groups around Brisbane, Australia and a research network of over 30 academics. Two films covering the project have been produced by Brightmoon Media. His work has been covered on the BBC One Show and Al Jazeera and in The Independent and ABC. He has regularly contributed commentary to national and international media and is BBC Election Night analyst for BBC Radio Cumbria. He is founding Editor of Global Discourse, a quarterly Bristol University Press journal.

3 Victoria Gallagher is a Community Organiser, working to engage and support individuals, groups and organisations in community participation and to develop projects designed to change communities for the better. She was centrally involved in 'A Cross-cultural Working Group on "Good Culture" and Precariousness' and has delivered material as part of undergraduate and postgraduate modules at Lancaster University.

${ }^{4}$ Roger Appleton has been making films for 20 years. Originally making pop videos and then moving into factual film production. He has extensive experience of making factual films for BBC2, BBC4, Sky Arts, Granada. This has included work with both internationally successful musicians and actors, high profile politicians, artists, and cultural commentators and people from excluded and vulnerable communities. He produced two films on 'A Cross-cultural Working Group on "Good Culture" and Precariousness" and has delivered material and training as part of undergraduate and postgraduate modules at Lancaster University.
} 
action. PAR uses a range of different methods, both qualitative and quantitative. (Participatory Methods 2018)

The method is grounded, centrally, in the creation of collaborative research relationships between academics and communities, informed, epistemologically, by concern for knowledge of the local, in the development and production of research aimed directly at understanding and responding to issues of importance to both parties.

At a time in which disaffection with politics is growing, there is great scope for PAR to be deployed by political scientists to examine a wide range of phenomena and, importantly, to advance collective responses that make sense to those at the heart of myriad challenges. PAR offers the potential for those in the field to make use of both inter- and multi-disciplinary resources and the knowledge and skills of non-academics to develop much more holistic accounts and to build political action through research. The problem, though, is that the method is often misunderstood and regarded as either unacademic or too unwieldy to deploy. It is essential, therefore, that the possibility of deploying such approaches is disseminated clearly, meaningfully and effectively to Early Career Researchers (ECRs).

In this article, we identify a series of core challenges in teaching PAR and present a series of means of dealing with these challenges pedagogically in such a way as to enable relevant ECRs' to adopt the approach. In keeping with participatory approaches, we then work through reflection upon co-delivery of FASS644: Participatory Methods: Achieving Impact in 2017-2018. The module is part of Lancaster University's Faculty of Arts and Social Sciences Postgraduate Research Training Programme (FASS RTP), which enables all research postgraduates and some taught postgraduate students to apply for places on modules, and is codelivered by a Senior Lecturer in Politics, Matthew Johnson, an experienced community researcher and Community Organiser, Victoria Gallagher, and an experienced filmmaker, Roger Appleton, of Brightmoon Media, who has worked with community groups to document their lives as part of a broader participatory project. We outline the content of the module to provide colleagues teaching methods with means of developing sessions grounded in their own specific needs. We then reflect, in light of participant feedback, on the effect of the teaching on participant approaches to the topic. The point is not to coerce participants into applying the methods, but to encourage those who can to do so, and dissuade, by raising awareness of the difficulties, those who are not in a position, for whatever reason, to do so effectively. By outlining the content of the module below, we also provide an introduction to PAR for colleagues attempting to advance critical methodological approaches to a range of pressing issues. While we refer to the UK context by way of illustration, the themes, pressures and capacities attendant to the article are apparent globally and the practice we share can be adapted for application within myriad different contexts. This is important as PAR presents political scientists with means, not just of understanding power, authority and politics in its broadest sense, but of building political action, specifically among excluded and alienated groups.

\section{The challenges of PAR}

Historically inspired by Marxist, feminist, critical theoretical and anti-colonial thinking, the method is now deployed for a range of ethical, but also practical, reasons. Whereas traditional academic work focuses on research on communities, participatory approaches work with and for communities. In this respect, the defining feature of PAR as a method is the creation of egalitarian, mutually supportive relationships to the furtherance of an end. It is for this reason that we refer to method in the singular, rather than methods in the plural, since, although the research produced necessarily differs radically by virtue of the local, issue-specific nature of projects, it is the creation of the relationship between different actors that is afforded central value. Actions that stem from that relationship include: co-production of decisions about the 
research topic, methods and questions; performance by community members of research/fieldwork, with training and support from academic researchers, and co-production with communities of analyses, reports and other research outputs, leading to myriad impacts (Durose, Beebeejaun, Rees, Richardson and Richardson 2012).

It has been used widely in public health (see Horowitz, Robinson and Seifer 2009), where attempts are made to satisfy the need of policy makers and communities to understand reasons for ill-health and the need of people themselves to promote their own health. Its applicability is much broader, though, and researchers are increasingly exploring opportunities in a range of other fields (see O'Toole et al. 2003: 592). This trend is influenced by the emergence of the impact agenda that increasingly underpins evaluations of research and plays a significant role in the allocation of jobs and grants, as illustrated by the UK Arts and Humanities Research Council's (AHRC (2018) Connected Communities Research Grants Highlight for Connected Communities and Design.

There are good reasons, therefore, for Early Career Researchers (ECRs) to understand approaches aimed directly at facilitating engagement and change as an integral part of research. However, scholars are often unaware of the potential, nature and implications of the method and there are, as yet, few formal opportunities within institutions to enable scholars to develop competence. Where there is awareness, the approach is often seen as too complicated, timeconsuming or impractical, particularly for ECRs, while, in disciplines dominated by traditional, library-based research, it is often regarded as irrelevant or unacademic. Quite aside from the social value conferred by participation, it ought to be recognized by those academics that such collaboration increases the chances of projects' being funded, recognized and/or endorsed by virtue of emergent impact agendas while, as myriad examples from public health demonstrate, there is good reason to believe that participation can lead to relevant, academic outputs. As such, communities and academics have a mutual interest in collaboration (see Bergold and Thomas 2012) and ECRs in evaluating the possibility of adopting elements of the approach.

While there is a significant literature on the approach itself (see Kemmis, McTaggart and Nixon 2013) and many different attempts to reflect on specific experiences in projects, there has been less discussion of approaches to teaching the method to this new generation of researchers by drawing on materials and resources from a participatory project. McNicoll (1999) has come closest to addressing this issue, asserting six challenges in such teaching: '(1) the need to adopt a new research perspective; (2) the tension between grading and modelling; (3) the realization that ethical considerations sometimes hide social control elements; (4) the limitation of the time frame; (5) the tension between research and action; and (6) the need to pay attention to group process'. We do not dismiss such claims. Rather, we argue that the challenges of disseminating the method to ECRs differ from those of McNicoll's undergraduates, that McNicoll's concerns can be accommodated within a different structure and that the context of research has changed as a result of pressures within Higher Education. We argue, instead, that the challenges to address with ECRs today are:

i) demonstrating the intrinsic value of the approach;

ii) emphasizing the difficulty of communication between different actors;

iii) explaining the limitations of traditional models of ethical review processes and 'consent';

iv) evaluating the feasibility of securing outputs compatible with sustaining a career in academia, and

v) facilitating means of enabling ECRs to articulate projects in such ways as to attract grant funding that is often more elaborate than that required for traditional projects (see Bennett and Roberts 2004, 7; 22). 
Before we outline our responses, we wish first to note the methods that we have used to present responses to these challenges.

\section{Note on method}

Reflecting the nature of the research project from which the teaching is drawn, this article is grounded in a practical action method deployed to 'solve a practical problem' and 'produce guidelines for best practice' (Denscombe 2010, 6). While we acknowledge Lethwaite and Nind's $(2016,413)$ assertion of a prima facie benefit of shifting 'the focus from individual experiences of research methods teaching to empirical evidence from a study crossing research methods, disciplines and nations', we do not share their belief that enhancing 'pedagogic culture and practice' ought always to stem from 'analysis of expert responses to the distinct pedagogic challenges of the methods classroom'. In certain respects, the focus on expertise rather than experience runs contrary to the very underpinning commitment of PAR to disabuse actors of their status as experts and to recognize knowledge as grounded locally in particular sites. Indeed, while Kilburn, Nind and Wiles $(2014,204)$ are surely right to promote 'empirical investigation beyond practitioners' reflections on particular courses or of contexts', given that teaching of PAR to ECRs is still in its infancy, that empirical investigation can only proceed upon a foundation of experience-sharing.

As such, this article's contribution to understanding critical methods stems precisely from belief in the value of case study: 'an empirical inquiry that investigates a contemporary phenomenon within its real-life context; when the boundaries between phenomenon and context are not clearly evident; and in which multiple sources of evidence are used' (Yin 1984, 23). The research presented here is an intrinsic case study advanced to understand and explain the pedagogy of participatory research for its own sake (Stake 1995) and in its own context (Mann 2006, 78) through 'worksite' engagement (see Denscombe 2010, 134). We present this account of practice as the basis for colleagues' development of their own teaching on PARs around which further interdisciplinary pedagogical research can be advanced. The account attempts to demonstrate means of 'Engaging learners in the research process, providing handson experience and offering opportunities for critical reflection' which, for Kilburn, Nind and Wiles $(2014,203)$, 'constitute important complementary and overlapping elements of... [a] formally articulated pedagogy' grounded in the reflection of teachers on practice. The research serves, therefore, the needs of those attempting to advance programmes of teaching participatory research through considered illustration and reflection on a particular experience. Such an approach, which combines practical action research and case study in fostering critical action research, has natural synergies with participatory methods insofar as the latter involves collaborative transformation of people's lives (see McAllister and Vernooy 1999, 48-59; Freire 1973).

In this respect, it is important to note that 'best practice' will differ from institution to institution and subject area to subject area according to the material deployed to illustrate the teaching, but that the fundamental issues with which to engage are fairly consistent. The way in which those issues are advanced must necessarily respond periodically to the student 'feedback loop in which initial findings generate possibilities for change which are then implemented and evaluated as a prelude to further investigation' (Denscombe 2010, 126). The approach adopted here conforms to the notion that action research is more of a strategy than a specific method (Susman and Evered 1978, 589) and draws reflexively upon a questionnaire completed by the first cohort of doctoral ECRs to undertake the module as means of reflecting on impact within this particular context. This questionnaire consisted of an explanatory section, a quantitative section examining the extent to which the module enhanced understanding of elements of participatory research methods and a qualitative section of open-ended questions examining the impact of learning about such methods on participants' thinking about their own 
research. The research was conducted during the development and implementation of the module by us (as academic practitioners, community workers and media professionals), on our own teaching practice as we were engaged in it (Edwards and Talbot 1994: 52).

Before outlining the module's content and reflecting upon the module's first iteration, it is necessary to explain the research context from which the pedagogical approach developed.

\section{Practice context}

The Conservative-led UK Coalition Government of 2010-2015 focused centrally on providing a neo-liberal response to a neo-liberal problem. The Conservatives claimed, centrally, that the commitment to a stripping back of the state was, both, ideologically coherent and the prudent response to indebtedness stemming from the bailing out of banks and the loss of tax revenue. In 2010, this commitment to small government was attached to a 'well-being agenda', which sought to evaluate policy according to its contribution to well-being, and David Cameron's flagship 'Big Society' initiative, which sought to devolve social action to civil society. This approach challenged communities stripped of state funding to take responsibility for their collective interests at a time in which concern was rising about the insecurity or precariousness of modern lives (see Standing 2011). Having advanced cross-cultural means of evaluating culture or shared understandings on account of the extent to which they promote well-being within a particular context (see Johnson 2013) and taken note of arguments against intervention in communities (see Wall 1998), we sought, in 2011, to explore the extent to which groups most disadvantaged by contemporary socio-economic policy could actually make use of the space afforded by 'Big Society' to advance their collective interests.

We sought to do this through a participatory project involving people from communities deemed dysfunctional by their respective Governments, but which had appeared relatively functional before neoliberal understandings came to underpin socio-economic policy: Ashington, Northumberland, a former coal mining village, and Aboriginal groups around Brisbane, Australia (see explanation in Johnson 2017). In this context, ten non-academic community co-researchers, five from Ashington and five from Brisbane, spent two years developing relationships with one another in advance of one-month embedded visits to each other's communities in mid-2015. During these exchanges, the co-researchers lived in their counterparts' communities, shadowed their hosts in their workplaces and social environments and conducted research on their (often overlapping) areas of interest: relationships, employment, health, education, environment and arts. The groups engaged in interviews, focus groups, daily group meetings and weekly seminars as they developed their ideas. Interviews, presentations and discussions were recorded on film during the production of two documentaries on the project by Appleton. There then followed three British Academy Rising Star Engagement Award (BARSEA) conferences, each aimed at explaining the lives of group members within the context of their deep history and outlining means of developing meaningful collective responses to challenges with the participation of academics and community professionals from a range of backgrounds and disciplines. The interviews, discussions, documentaries and conference presentations are all available on a designated project website (Johnson 2017) and YouTube archive (Johnson 2018).

The project provided the groups with the structure to think collaboratively and politically about the ways in which their traditional cultural resources might assist them in dealing with challenges in the present. The participatory engagement and co-production with academics was intended specifically to produce rich, detailed and coherent accounts of life within seriously disadvantaged communities (see claim with regard to poverty in Bennett and Roberts 2004). We drew upon this experience of collaboration, in particular, to inform, shape and illustrate the teaching within the module and to provide a pivot around which to refer participants in discussions. 


\section{The module}

The structure of the module was constrained by the numerous scheduling complications attendant to doctoral students' employment, teaching and research commitments that extend beyond those of undergraduates. Such constraints apply also to the teaching staff, each of whom has a different set of commitments concomitant with their professional and personal lives. As such, despite McNicoll's (1999, 57-58) assertion that participatory research requires significant investment in time, in our case, it was inevitable that contact hours were condensed into single days. We decided to restrict teaching to: a workshop day consisting of four 90 minute sessions; assessment of a 3,000 word research grant proposal on which formative feedback is provided two weeks after the workshop day, and a two hour-long peer-feedback session a further two weeks later. Teaching roles are allocated to reflect the particular expertise of the contributors. All of the lecturing and plenary discussion was filmed in its first iteration and uploaded to the project YouTube archive to be embedded into the module online learning site, along with session PowerPoint slides, all readings and other course documents. The first four workshop sessions each contain 45 minutes of lecture and 45 minutes of activity. Because of the innovative nature of participatory methods, the content of the module is heavily focused on illustration through reference to real world examples. In the workshop day, participants develop material for their mock grant proposal to fund a prospective participatory project grounded in their own work and then revise that proposal in the feedback session. The point is both to enable participants to ascertain the viability of adopting such methods and to take practical steps towards advancing such a project if it appears viable. Each of the sessions relate specifically to one of the five challenges we note in the introduction.

\section{Session 1: Introducing Participatory Methods: Origin, rationale and application}

The first session seeks to outline the compelling reasons for the method, of which there are many:
The rationale for using participatory research may be functional, to encourage community participation in order to improve the usefulness of the research to local people. For example, to help develop farming technologies more suited to the local area and needs or to improve reach and speed of adoption of new methods and technologies. Another reason may be for empowerment or social transformation, to strengthen local people's capacity in decision-making, in research, and in management of local resources, in order to improve their awareness of options and to strengthen their ability to act on their own behalf... Often participatory research is both functional and empowering. (McAllister and Vernooy 1999, 8)

For academics, such methods can offer more complete forms of research with richer findings that speak to effects on human lives (see Fox, Suryanata, Hershock and Pramono 2004) and outcomes with genuine impact (see Kelly, Mulgan and Muers 2004). Indeed, buying into participation can foster transformative approaches to principles of research through the development 'research governance frameworks' (Beebeejaun et al, 2014b) that outline 'principles of good governance that apply to all research within the remit of' particular institutions or organizations (Department of Health 2005: 8).

Perhaps most importantly, experience of participatory research is transformative insofar as it leads academics reflexively to reconsider the place of the academy and its activities within society (see McNicoll 1999, 55). It can help to challenge imbalances in power (see Pain, Whitman and Milledge 2011, 5-6) apparent in other methods and facilitate collective community agency as groups examine issues of importance to them. While there is 
acknowledgement that academic and non-academic participants have different roles in research, there is explicit recognition of value pluralism, with the knowledge, expertise and contribution of different parties regarded as necessarily incommensurable and, often, indispensable (see Heart and Wolff 2006). We draw upon our own diverse skill sets to highlight the importance of recognizing value pluralism in practice. We argue that academics have the capacity to explore and understand issues and to articulate those understandings in important ways and that academic involvement can add institutional accountability (see Silverstein, Banks, Fish and Bauchner 2008, 70) to forceful and engaged community positions, enhancing the capacity of groups to secure resources from non-academic sources that often require findings that can only be produced by specialists (Durose et al, 2012). Participation means, though, that these findings must be articulated in ways that can be understood by those who have often been alienated by academic discourse, including through photography, videography and non-academic prose (Beebeejaun et al. 2014a).

To foster understanding of the rationale of PAR, we ask participants to work individually for 10 minutes to identify a) the core assumptions that they make regarding their positions in society and the value of their research to society, b) the non-academic skill and knowledge sets that would benefit their research, and c) the particular non-academic partners with whom they wish to work in a prospective research project. Participants are then placed in inter-disciplinary pairs, taking turns over 15 minutes to present their assumptions and pitch their need for participation to their partner, who attempts to adopt the perspective of the nonacademic partner with whom they wish to work. Participants then consider individually, over 5 minutes, how their view of their research might be affected by working with non-academic communities. In the final 15 minutes, we examine some of the core assumptions as a group, attempting to identify opportunities for making best use of academic skills and complementing those skills and forms of knowledge with specific non-academic skills and forms of knowledge. The purpose, reflexively, is to enable participants to consider the radical, transformative potential of participatory research.

\section{Session 2: Preparing: identifying partners and developing a shared language}

The second session builds upon the transformative claim of the first session to enable participants to consider means of building relationships with non-academic actors. Here, it is suggested that the first contact between academics and communities is often made by researchers with existing project proposals that require community participation or by communities with specific projects that require specific forms of academic expertise (see discussion in Heart and Wolff 2006) or evaluation (see Pobal 2015). Increasingly, however, there are academic forums that actively solicit requests for participation from communities. These include the Community-Academics Research Links (CARL) (2017) at University College Cork, which enables organizations to engage with students (undergraduate or postgraduate) who carry out the research under the supervision of an academic supervisor (see O'Dowd 2016), and the Participatory Research Hub at Durham University (2017). These programmes offer communities opportunities to engage with academics from a range of different disciplines and with a range of different skills on a relatively low cost or fee free basis.

However, it is clear that most first contact between academics and non-academics is academic-led. As such, it is essential that academics are well-placed to articulate the need and value of research in ways that make sense to non-academics. Getting that first contact right enables a much clearer set of discussions about whether and how best to take the project forward, what form participation will take, what findings or outcomes are to be pursued and how the research will be articulated. Of course, the greater the level of participation envisaged, the more open-ended this initial contact, leaving greater scope for the prioritization of ends by community members. This all requires academics and community members to develop a shared 
language in which clear, mutually beneficial agreements can be made and upheld. The process of shaping that language is inherently transformative, both for academics and communities, challenging power imbalances surrounding the attribution of 'expertise' and 'expert language' to particular parties.

To illustrate these concerns, we draw first upon the work of Siobhan O'Dowd and Denis Barret (2017), who converted a co-produced textual guide for communities engaging with academics into a visual guide designed with the needs and interests of the target audience at hand (Gallagher, et al. 2017). We then draw upon the example of Valdimar J. Halldórsson (2017), who traces his attempt to develop a project aimed at improving health and social care service provision to children with disabilities in the Westfjords of Iceland. He describes his fraught attempts to secure and sustain the support of sceptical local NGOs and an epiphany experienced while being confronted by parents of those children whose everyday struggles pose obstacles to participation. He argues that participatory projects call for deep intersubjective understandings of the challenges faced by people within specific contexts and a need to identify means of achieving credibility at the very outset of engagement.

To apply the general principles from these cases, we ask participants individually over 15 minutes to develop an embryonic project description detailing in non-academic language, a title, topic, description, background, aims, methods, expected outcomes and timetable. Participants are asked, in developing this description, to consider the following questions (which have similarities to those in other guides, such as Pain, Whitman and Milledge 2011, 4; Gallagher, Johnson, O’Dowd, Barret, Richardson and McNally 2017):

1) What is the reason for contacting the community? Does the community have specific collective resources, such as a shared identity or experience, or interest that enables a project to be developed? It is essential that the reasons for contact are made clear so that communities can understand the impact of the research on their individual and collective interests (see discussion in Silverstein, Banks, Fish and Bauchner 2008, 69).

2) What interests do people hold within the community? It may be that people have attachments, goods or principles that go unsaid, but which need to be explored.

3) What interests might be affected negatively by the project? While it may be that the project is of benefit to all, the project may also, unintentionally, promote harm for example, through the deployment of funding from a particular body (see Ball and Janyst 2008, 34).

4) To what extent can communities collaboratively shape the research and the way in which that research is conducted? This question speaks to ethical as well as logistical considerations, raising shorter- and longer-term implications that go beyond the lifespan of the project (see Banks and Manners 2012, 8).

5) What training, if any, will be required for community members to engage in the project? It may be that community members' existing expertise, with regard, for example, to cultural knowledge, is sufficient for the project to proceed. However, individuals may, again for example, require research training with regard to data collection, creating opportunities for capacity building as a means of value and knowledge exchange within the project (see West 2015; Ball and Janyst 2008).

6) How will the research be communicated in ways that the communities understand? This is of great importance in terms of impact, both in the sense of affecting the thinking of particular actors and in the everyday sense of the effect of the research on people's lives.

7) How might participation be recognized? Academics are attuned to recognition through publication and authorship, but there may be many other means of recognition 
both in terms of research outputs and the formal allocation of positions, which may be of significance among disadvantaged groups that have to grapple with welfare and employment considerations (see discussion in Heart and Wolff 2006).

Participants then work in their pairs for the next 10 minutes to present and highlight the (often unconscious) use of unexplained jargon and to identify problematic assumptions with regard to answers to the questions above. We then draw upon the feedback as a group, in the remaining 20 minutes, to identify general issues of intelligibility and assumptions about power relations between parties.

The session is grounded in making clear to participants the inherent diversity of positions, interests and possible contributions within projects (see Bessette 2004, 33-37). It emphasizes the intersubjective nature of collaboration and highlights the necessity of clarity in language to relationships of trust between academics and non-academics. This is the basis for any productive, mutually beneficial project. Completing the exercise enables participants to begin to recognize the power parties hold within the prospective relationship, fostering comprehension of some of the practical, professional and personal impacts that collaboration can create and leading to appreciation of the importance of sound ethical agreements.

\section{Session 3: Processes: ethics and governance}

Participatory ethical approval procedures and agreements are often significantly more complex than those in traditional researcher-subject arrangements, insofar as they appreciate the social, relational, interdependent nature of human beings and are informed by commitments to values of equality, consensus and collectivism (see examples in Banks and Manners 2012, 8). Because the approach recognizes the equality or incommensurability of participant roles and diversity of interests among parties, far greater weight is afforded to the input of non-academics to developing agreements (see McNicoll 1999, 56). Moreover, given that projects are much more likely to be more open-ended and to involve ongoing discussions as to the development of research, it is clear that agreements have periodically to be made and remade (see discussion in Chevalier and Buckles 2008, 39). More ambitious, co-produced projects are more likely, therefore, to adopt staged ethical agreements in which participants consent to each specific action, developing new agreements as new stages to the research emerge and the implications of those stages become clear. The purpose of this session is to illustrate the nature of agreements in such a way as to enable participants to identify the limitations and constraints of consent.

To begin, we introduce the Thames Valley Police's (2015) video on consent to sex. The video, which is accompanied by a trigger warning, was developed with survivors of sexual assault and has been widely welcomed for its innovative and powerful means of demonstrating the simplicity of consent. The video demonstrates the incoherence of apologists of breaches of sexual consent by invoking the example of giving someone tea. At each stage, excuses for breaches of sexual consent, such as prior behaviour or earlier agreement within a different context, are ridiculed through matchstick illustrations of people pouring tea into one another's mouths while actively resisting or unconscious. The point is that consent consists of the capacity of an individual to refuse participation. If that capacity is withdrawn, through unconsciousness, no consent can be attested. The video also shows that the actions to which individuals consent change swiftly and unpredictably in ways that make the original provision of consent irrelevant. In effect, the video suggests that, in many areas of our lives, we give staged consent to actions. This highlights the central difference between traditional forms of research that focus, ethically, on respect for persons in the Kantian tradition, and participatory forms that focus, in the republican tradition, on non-domination and the capacity of participants to say 'no' (see Pettit 1999, 165). 
We then demonstrate the complications of consent within a participatory project through reference to "A Cross-Cultural Working Group on "Good Culture" and Precariousness'. Within that project, there were several stages. First, there was a preparatory phase in which the participants developed relationships, shaped the context of research and identified practical considerations of their work. There was then an exchange phase, in which the community members, accompanied by an academic, visited each other's communities. This involved participation in professional and community shadowing roles as well as engagement with organizations in a research context. Alongside this, there was a data gathering and documentary making process in which the actions and thoughts of the participants were recorded by themselves and a filmmaker. Finally, there was a dissemination phase, in which the participants were joined by academics, community professionals and politicians from a range of backgrounds in three conferences and a series of associated exhibitions and events, leading to release of several documentaries for broadcast on television, a video archive, a series of op-eds and an academic journal special issue. Even in this cursory overview, it is apparent that there were several categories of participants (community members from the two communities, academics, community professionals, documentary makers) each with different roles and often competing interests.

In order to demonstrate the complexity of the process, we focus on the staged production of the documentaries. This was particular contentious given the recent context of 'benefits porn' series, such as Benefits Street, and the way in which the media is seen to have a particular vested interest in producing titelatory material with long-lasting impacts on the sort of communities at the heart of the project. To produce an ethically appropriate process through which the documentaries were produced, we agreed that the project participants both in the UK and in Australia would be part of the filmmaking process and would agree to the release of the films only once editing to their satisfaction was completed. This right of veto, highlighted in the Thames Valley Police video, was to ensure that the film does not compromise the interests or professional codes of conduct of the contributors. In addition, as part of the transformative principle of participatory research and the knowledge exchange element of funding, Appleton provided filmmaking training to the community co-researchers, which fostered capacity to understand the production process and to take part, not just as 'subjects' of film, but as makers of the films, by gathering material themselves and contributing to the editorial process, specifically with regard to their portrayal. We agreed, finally, that any revenue produced from sales to TV or educational institutions would be split equally between the filmmaker, the Ashington community group and the Aboriginal Australian community group and channelled accountably through organizations with charitable status. In order to ensure compliance, Fiona Aiken, Secretary of Lancaster University, agreed to act as Independent Arbitrator for the documentaries in case of any dispute between the parties in the development, filming, editing, production and distribution of the documentaries. This meant that there was an ethical structure to the project that enshrined the principle of staged consent, upheld people's interests and enabled open-ended research to take place.

To apply this general and illustrative discussion to individual projects, participants are asked to draw upon their answers from the previous two tasks to produce, over 20 minutes, a list of 'risks' for community participants in speculative chronological order of their emergence, a set of associated stages in which those risks might be identified and managed and a series of organizations or parties that might have existing ethical codes to deal with potential issues of duty of care during the project. These overviews are presented for critical feedback from partners over the following 10 minutes before a plenary session in the final 15 minutes in which general risks and strategies are noted from the separate discussions within the cohort.

Session 4: Outputs and outcomes: Recording, documenting and publishing research 
Given that academic careers are increasingly grounded in such research outputs as articles in high ranking journal or books published by leading university presses, it might seem counterintuitive to invest time and effort in producing work with non-academics who may lack the ability to create such publications. Indeed, one of the key aims of the module is to emphasize to potential users of participatory methods the necessary investment of time and effort that may detract from more instrumental publishing strategies (see Ball and Janyst 2008, 34). However, there are ways in which the approach is instrumentally, as well as intrinsically, valuable. In particular, there is scope for production of research that is richer by virtue of the investment of community participants, providing rounder accounts and explanations for phenomena than is possible using other methods (see Flicker, Savan, Kolenda and Mildenberger 2008). It is also more conducive to meaningful, measurable impact (Catley, Burns, Adebe and Suji 2014, 2025). Moreover, such forms create scope for much greater participant input and ownership than other, more traditional forms of dissemination. Indeed, such participation is increasing a criterion of funding and is more likely to lead to further funding. As Research Councils and Funding Bodies move to use impact as a metric of evaluation of proposals, researchers need to be aware of the ways in which the investment of time and effort incumbent in participatory methods may come to be seen as a base line for funded research (see discussion in Bennett and Roberts 2004, 25-26). How, though, should that time and effort be invested? We focus on four main possibilities.

The first concerns conferences or workshops. These offer transformative interaction between academics and non-academics in sites that either cohort may not previously have experienced. In our project, we held one event on campus at Lancaster, bringing community co-researchers and members of the research network to the North West for a weekend. This was particularly useful insofar as several non-academics had not previously entered or stayed on a university campus. Participating in the event in that site helped deconstruct misconceptions about Higher Education institutions and feelings of alienation from education (see discussion in Heart and Wolff 2006). We then held a second event in the UK project site, Ashington. We used The Ashington Community Development Trust space within the former Co-Op building, which has a large hall for meetings and events. This setting helped to enable research network members, particularly from overseas, to grasp the seismic changes inflicted on the community, with a once thriving site of industry, commerce and socialization now reduced to a vast, empty, decaying and, on the weekend in question, freezing monument to the village's past. The experience ensured that the community members had ample means of demonstrating their challenges to those with little prior knowledge of the area, fostering a sense of understanding that may not have been possible had the conferences been held solely on university campuses. At each event, participants were introduced solely by their first name and by their self-identified place of origin, stripping back the formal means of distinguishing roles. This ensured that discussion shifted in nature and reduced the capacity for domination of discussion by academics. We argue that such forms of public dissemination are illustrative of the sort of transformative potential of participatory approaches.

Second, we argue that a designated website with associated audio-visual materials is an essential component of contemporary participatory projects. Even basic sites can act effectively as a hub for the project, a reference point for potential collaborators and a point of dissemination as outputs emerge. To foster broader dissemination, the talks, workshops and discussions from our conferences were filmed, edited and uploaded to a dedicated YouTube channel by Appleton. The videos, which are embedded throughout our project site (Johnson 2017), act as a free online resource for researchers, ensuring that full value is gleaned from the events. Increasingly, those who deploy participatory methods regard such means of dissemination as necessary features of projects and there are many existing sites that outline and promote participatory research (such as Participate: 
http://www.participate2015.org/about/) and track researchers' experiences (such as University of York's Participatory Research blog: https://participatoryresearchblog.wordpress.com/). We outline the way in which a site capable of reaching those within and beyond academia can be developed simply, often through a university's associated WordPress facility. We argue that, with a functioning ethical protocol, researchers can engage with individuals effectively and productively through video (see Bessette 2004, 64), collating qualitative data effectively with rudimentary equipment and producing public records through uploads to video streaming sites, such as YouTube and Vimeo. We highlight the use of our (Johnson 2018) archive as a resource for use and embedding in teaching and research materials. Beyond that, we argue that there are large numbers of filmmakers capable of assisting professionally in the project with direct interests in community work and, in some cases, the specific communities involved. This provides unprecedented scope for documentary making, offering forms of communication previously restricted to narrow, well-funded commercial and public broadcasters.

We then consider policy papers for development with community organizations as a means of engaging with think tanks, local government and government departments (see discussion in Tandon, et al., 2007, 6). We argue that increasing reliance upon public engagement as a means of demonstrating impact and accountability ensures that a range of previously aloof bodies are now willing to involve clearly articulated participatory projects in the development of publications and programmes (see Shore, Wong, Seifer, Grignon and Gamble 2008). This means of fostering impact is enhanced through production of co-authored or group-authored op-ed pieces for publication in the mainstream press, online media and research body and funding council periodicals. We trace associated publication processes, highlighting the importance of facilitative Press Offices and the value of forums such as The Conversation as means of achieving subsequent publication in the mainstream press. We then examine often unpredictable and chance engagement with policy makers through the example of an op-ed in ESRC Society Today leading to correspondence with a Liberal Democrat Member of the House of Lords.

Finally, we introduce means of evaluating prospects for co-authored or group-authored academic publications in journal article, edited collection and monograph form, which are discussed by Bordeaux et al. (2007). We illustrate means of fostering dialogue between academics and non-academics in traditional academic forums through the example of the project's special issue of Global Discourse (7:4) on 'Precariousness, Community and Participation', which featured extended replies to articles by community co-researchers and community professionals. The special issue, which was developed from contributions to the final project conference, has been published on the project website using author copyright entitlement to use pre-production open access versions of articles. This, we argue, offers means of drawing upon the different skills of participants, introducing transformative dialogue to academic forums, ensuring professional recognition for academics and facilitating broad, open access, dissemination. Such special issues are often natural means of codifying contributions to other project forums and rounding off phases of research effectively.

We ask participants individually to spend 15 minutes identifying i) an element of the research that can be filmed and that will provide value to others by being disseminated online; ii) a policy area that could be examined through a policy paper and a suitable organization to which the document could be sent; iii) two prospective contributions by a non-academic coresearcher to an academic journal article and two particular obstacles, either ethical or practical, to those contributions being made. Participants work in pairs over 10 minutes to present findings and provide feedback with regard to their feasibility. We then evaluate as a group in the remaining 20 minutes the possible effect that pursuing the publications identified will have on participants' respective careers in comparison to traditional approaches to publishing. 
Finally, we introduce the assignment by outlining a set of key principles around which all grant proposals ought to be grounded. For this, we draw upon the UK Economic and Social Research Council's (ESRC 2018) guidance, which emphasises the necessity for need, relevance, appropriateness, feasibility, cost-effectiveness and excellence. We then clarify the nature of impact through reference to the Higher Education Funding Council for England's (HEFCE 2016) definition for REF Impact Case Studies: 'an effect on, change or benefit to the economy, society, culture, public policy or services, health, the environment or quality of life, beyond academia'. We emphasise that, in general, such impact ought to stem from peerreviewed articles, monographs and book chapters, before highlighting the way in which sites of knowledge exchange, such as those outputs listed above, can stem from those academic publications. This creates a framework around which participants can complete their assignments.

\section{Independent Study}

In order to apply the material from the sessions, participants are given two weeks in which to produce a 3,000 word mock grant proposal based on the material they have developed within the first four sessions. Influenced by Kilburn, Nind and Wiles' $(2014,203)$ formally articulated pedagogy, the assignment is intended to provide 'hands on experience' of an integral academic activity of profound importance to the profession, in ways that engage 'learners in the research process' by drawing on their own work, and offer 'opportunities for critical reflection'. Participants are asked to identify a funding programme most suitable to their research. ${ }^{5}$

\section{Session 5: Feedback}

The final, two hour, session takes place four weeks after the workshop day. The session aims to foster and refine grant writing skills by providing general and particular feedback on the assignments submitted. In advance of the session, participants are asked to read through several examples of successful grant proposals and two examples of feedback on real grant proposals and are provided electronically with comments on their individual assignments representative of feedback produced by referees in response to real grant proposals. We spend the first 25 minutes of the session discussing general issues in the assignments, using the key principles as a reference point for guidance. Participants return to their pairs and discuss the applicability of the general points of feedback to their individual proposals for the next 15 minutes. Participants are then given 20 minutes to produce 3 minute summaries of their proposals revised in light of feedback from the module leader and their partner. These summaries are then pitched to the cohort in the final 60 minutes for provision of peer comments and further feedback. Participants leave the session having received written feedback on their specific proposal and general feedback from the module leader as well as the thoughts of peers from a range of disciplines.

\section{Reflecting on practice}

The module's first iteration faced several challenges. It was taken by twelve ECRs, at various stages of doctoral study, from subject areas throughout the Faculty of Arts and Social Sciences, including Politics, Sociology, Human Geography, Law, Design, Linguistics, Health and Psychology. Because participants take the modules voluntarily, and because departments do

\footnotetext{
${ }^{5}$ The headings from the mock proposal are taken from Research Council and Funding Body grant application forms of relevance to the Social Sciences, Humanities and Arts: Subjects; Abstract (200 words); Case for support: (1,000 words divided between the sub-headings as you see fit); Research questions or problems; Research context; Research methods; Technical Summary; Project management; Dissemination; Academic beneficiaries (200 words); Impact summary (300 words); Pathways to impact (500 words); Justification of resources (350 words); Workplan (200 words); Ethics (250 words). The space afforded under each heading is broadly representative of that afforded in grant applications, with disproportionate space afforded those sections relating specifically to participatory methods.
} 
not insist on participants completing those modules in order to present attendance of single sessions as evidence of fulfilling obligations to undertake research training during review panels, completion of assignments in the Faculty Research Training Programme (RTP) as a whole is low. Ultimately, despite the fact that participants drafted elements of the mock grant proposal during the workshop day, only six of the twelve participants submitted assignments. Although the $50 \%$ submission rate was deemed high relative to other modules, institutionally, the problem of assignment completion can only be resolved by departments' insisting that participants who volunteer to take modules as part of their doctoral study must complete those modules in order to be able to cite those modules in annual progress reviews. At module level, in future iterations, we will emphasise that the 3,000 word limit is just that - a limit - and that shorter pieces can be submitted for feedback. Indeed, on the basis of the first iteration, the rationale for encouraging submission is clear: those participants that did submit assignments demonstrated significant development of ideas from their first articulation during the workshop day. Two participants who submitted shorter pieces both developed cogent ideas around which projects could develop. We view this accommodating approach, in which feedback is provided on whatever work can be produced, as being in keeping with Kilburn, Nind and Wiles' (2014, 203) 'formally articulated pedagogy'.

The results from the questionnaire both support the value of the teaching and demonstrate the limits of PAR as an approach for ECRs. Five of those participants that did submit their assignments attended the feedback session, along with one participant that had not attended the workshop day. The other participant who submitted an assignment was unable to travel due to the February/March 2018 UK weather warnings. Eight of the twelve participants completed questionnaires. The quantitative data (see Table 1) indicated strong support for impact of the module in terms of understanding the rationale, communicative content, ethical considerations and means of documenting and disseminating participatory research, as well as suggesting encouragement for the deployment of participatory methods. However, the data indicated that the module had a mixed effect on the intention of participants to deploy the approach, with three strongly agreeing that they would, three neither agreeing nor disagreeing and two disagreeing. This fits with the intention of the module to support those capable of engaging in participatory research and dissuading those who, in full possession of information on the approach, would not be willing or able to do so. One participant consistently disagreed with the statements and seemed generally dissatisfied, but then replied contradictorily, 'lots', in response to the questions in the qualitative section.

Table 1: Quantitative data from questionnaire 


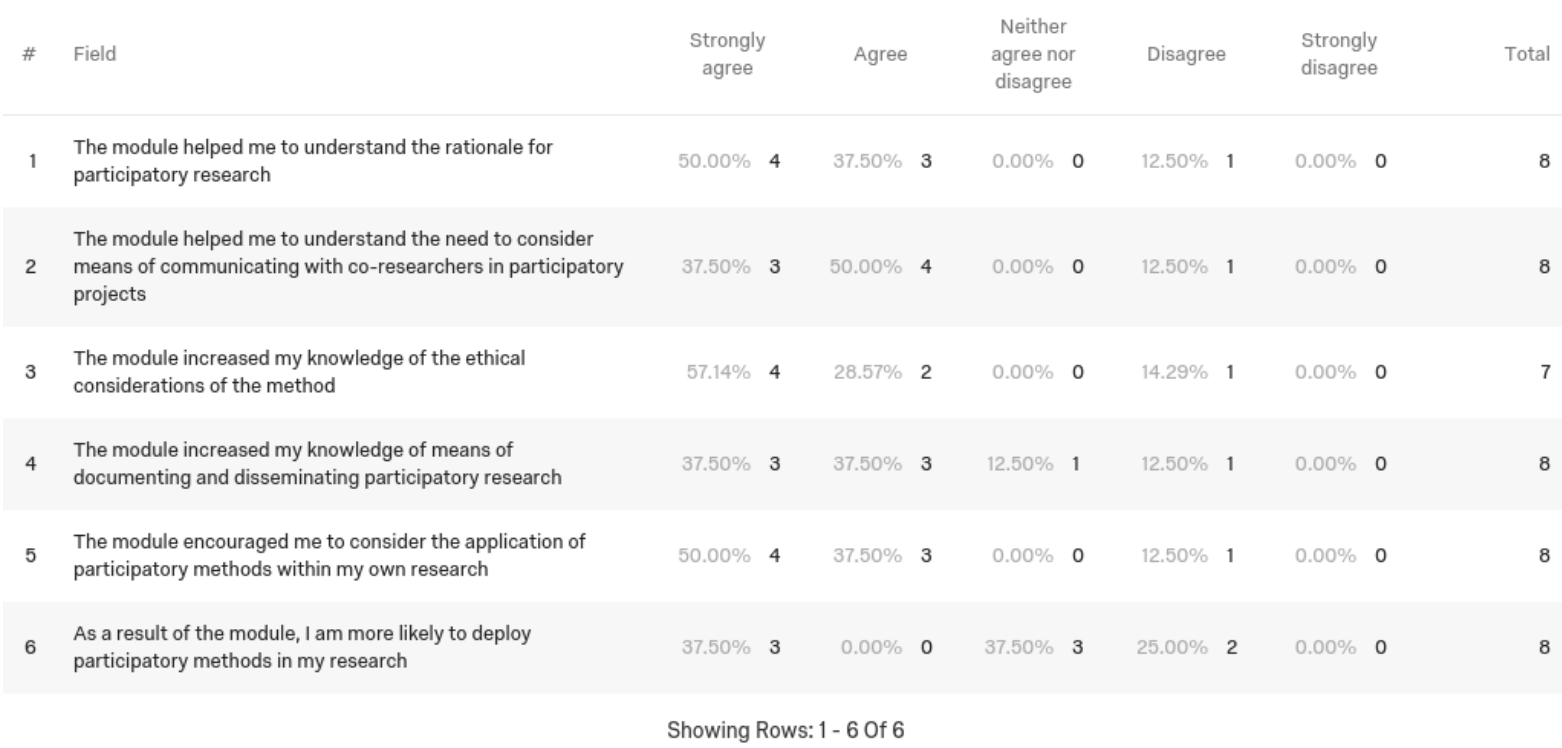

These findings were substantiated by the qualitative section, in which participants were asked to provide open-ended responses to questions relating to the topics of the ordinal questions. Respondents provided a range of thoughts on impact on self-understandings of the nature of research, knowledge and the role of researchers. Participants suggested either that positions and beliefs were reaffirmed or that awareness of power disparities and ignorance of subject groups in field work were raised, reflecting the fact that some participants already intended to deploy participatory methods. In terms of the impact of the module on the way in which ECRs approach communication about research, respondents talked of broadening means of communication and tailoring forms to particular needs, specifically with regard to dissemination to non-academic groups. Respondents then talked, with regard to ethics, of raised awareness of the vulnerability of groups and the nature of that vulnerability, particularly in relation to data collection and use, as well as the possibility of deploying staged consent as a means of addressing more complex projects. The ECRs added that, in relation to forms of communication, the module encouraged them to talk publicly about their project to a number of audiences, to explore audio-visual means of capturing and disseminating data and to think more rigorously about pathways to impact. In terms of the impact of the assignment, those participants that did submit the assignment were universally positive, stating that the exercise was 'Very essential, it gives advice, information for your future career', that it 'Focused my attention on issues that I need to address and to work on', 'Helped immensely in improving my proposal' and 'helped me to consider focusing more some areas I overlooked'. Its importance was indicated by its timeliness: 'I have to create a project plan very soon so it will sharpen what I write'. With regard to whether, and in what ways, respondents would be more likely to deploy participatory methods as a result of taking the module, one stated that they were already using such methods, so were not more likely to do so, another four suggested that they had been encouraged to use the methods, including one by developing the mock grant proposal into a real one, while another two suggested that the module had raised awareness, but not sufficiently to enable them to deploy the methods, with one suggesting that the module, while 'a good introduction to the concept', 'did not sufficiently direct in how to' use the approach. The final question asked for further thoughts on the module, with one respondent emphasising the difficulty of completion of first year $\mathrm{PhD}$ students completing the assignment by virtue of their lack of experience and another suggesting the introduction of an additional follow up session to explore the practice further. 
The dearth of more substantive feedback on the deficits of the module is regrettable. In the next iteration, greater care to explain the practice of methods will be made, specifically with regard to additional examples, and more support will be given in the fourth session in order to prepare participants to complete the assignment. However, there seems to be good reason to view elements of the module favourably in light of the feedback, specifically in an ECR research training setting. Perhaps the key finding is that some participants were dissuaded from adopting the method. This is, in many respects, a positive outcome. Participatory methods ought to be deployed only by those with the capacity and willingness to develop, sustain and co-produce projects - all of which is difficult and time-consuming with myriad unpredictable implications for participants. Teaching practice that encourages those with the ability to conduct research using critical methods, but discourages those without, ought to be seen as beneficial for both students and non-academic communities.

\section{Conclusion}

Co-designing and co-delivering a module such as this is in the spirit of participation. The sessions are each intended to relate to the five pedagogical challenges outlined in the introduction. In reflecting on the module, we believe, both, that modules on participatory methods ought to be available to ECRs at Faculty and university level (not least because of the reflexive value of interdisciplinarity) and that the module developed presents a valuable, albeit challenging (by virtue of institutional considerations), model around which other approaches can be developed. We also believe, firmly, that the use of example as a means of shaping content ought not to be seen as intellectually deficient. PAR methods, by their very nature, call for deployment of core principles in different ways to suit particular relationships and circumstances. We present this article, and the materials attendant to it, in order to encourage others to explore means of developing teaching of critical methods appropriate to institutional context. We also hope that this encourages colleagues to share their own practice in order to foster open and constructive knowledge exchange in the spirit of the method. While we acknowledge Lethwaite and Nind's $(2016,413)$ concern for empirical research 'crossing research methods, disciplines and nations', this can only take place on the back of this work.

\section{References}

AHRC (2018) 'Grants Highlight for Connected Communities and Design', AHRC, Swindon: AHRC, <http://www.ahrc.ac.uk/research/fundedthemesandprogrammes/crosscouncilprogram mes/connectedcommunities/fundedactivitycrosscutting/highlight-notice/> [Accessed 20 February 2018].

Ball, J. and Janyst, P. (2008) 'Enacting Research Ethics in Partnerships with Indigenous Communities in Canada', Journal of Empirical Research on Human Research Ethics, 3(2): 33-51.

Banks, S. and Manners, P. (2012) Community-based participatory research, Durham: Centre for Social Justice and Community Action, Durham University.

Beebeejaun, Y. Durose, C. Rees, J., Richardson, J. and Richardson, L. (2014a) 'Public Value or Public Harm?', Environment and Planning C: Government and Policy, 33: 552-565.

Beebeejaun, Y. Durose, C. Rees, J., Richardson, J. and Richardson, L. (2014b) 'Beyond Text', Community Development Journal, 49: 37-53.

Bennett, F. and Roberts, M. (2004) From Input to Influence, York: JRF.

Bergold, J. and Thomas, S. (2012) 'Participatory Research Methods', Forum: Qualitative Social Research, 13: <http://www.qualitativeresearch.net/index.php/fqs/article/view/1801/3334> [Accessed 20 February 2018]. 
Bessette, G. (2004) Involving the Community, Ottawa: International Development Research Centre, <http://idl-bnc.idrc.ca/dspace/bitstream/10625/31476/33/IDL-31476.pdf> [Accessed 20 February 2018].

Bordeaux, B. C., Wiley, C., Tandon, D., Horowitz, C. R., Bohrer-Brown, P. and Bass, E. B. (2007) 'Guidelines for Writing Manuscripts About Community-Based Participatory Research for Peer-Reviewed Journals', Progress in Community Health Partnerships, 3(1): 281-288.

Catley, A., Burns, J., Abebe, D. and Omeno S. (2014) Participatory Impact Assessment, Somerville, MA: Feinstein International Center.

Chevalier, J. M. and Buckles, D. J. (2008) A Guide to Collaborative Inquiry and Social Engagement, London: Sage, pp. 38-72.

Community-Academic Research Links (2017) 'About CARL', University College Cork Website, <http://www.ucc.ie/en/scishop/ac> [Accessed 30 July 2017].

Cooper, C. B., Dickinson, J., Phillips, T. and Bonney, R. (2007) 'Citizen science as a tool for conservation in residential ecosystems', Ecology and Society, 12(2): 11 [online] URL: $<$ http://www.ecologyandsociety.org/vol12/iss2/art11/> [Accessed 20 February 2018].

Denscombe, M. (2010) The Good Research Guide, Maidenhead: Open University Press.

Department of Health (2005) Research Governance Framework, $2^{\text {nd }}$ edition, London: Department of Health.

Durham University (2017) 'Participatory Research Hub', Durham University Website, <https://www.dur.ac.uk/esrciaa/test/researchingtogether/hub/> [Accessed 30 July 2017].

Durose, C. Beebeejaun, Y. Rees, J., Richardson, J. and Richardson, L. (2012) Towards Coproduction in research with communities, Swindon: AHRC online, available from: $<$ http://www.ahrc.ac.uk/documents/project-reports-and-reviews/connectedcommunities/towards-co-production-in-research-with-communities/> [Accessed 30 July 2017].

Edwards, A. and Talbot, R. (1994). The Hard-pressed Researcher. London: Longman.

ESRC (2018) 'How to write a good grant proposal', ESRC, Swindon: ESRC, $<$ http://www.esrc.ac.uk/funding/guidance-for-applicants/how-to-write-a-goodresearch-grant-proposal/> [Accessed 20 February 2018].

Flicker, S., Savan, B., Kolenda, B. and Mildenberger, M. (2008) 'A snapshot of communitybased research in Canada', Health Education Research, 23: 106-114.

Fox, J., Suryanata, K., Hershock, P. and Pramano, A. H. (2004) Mapping Power', in, J. Fox, K. Suryanata and P. Hershock, eds., Mapping Communities: Ethics, Values, Practice, Honolulu: East-West Center, pp. 1-10.

Freire, P. (1973) Education for Critical Consciousness, New York: Continuum.

Gallagher, V., Johnson, M. T., O’Dowd, S., Barret, D., Richardson, J. and McNally, E. (2017) A Visual Guide for Communities Working with Academics on Participatory Research Projects, A Cross-Cultural Working Group on 'Good Culture' and Precariousness, $<$ http://wp.lancs.ac.uk/good-culture/files/2016/07/A-Guide-for-Communities-WWAcademics-V2.pdf> [Accessed 30 July 2017].

Haart, A. and Wolff, J. (2012) 'Developing Local "Communities of Practice" through Local Community-University Partnerships', Planning, Practice \& Research, 21:1, 121-138

HEFCE (2016) 'REF Impact', HEFCE, Bristol: HEFCE, $<$ http://www.hefce.ac.uk/rsrch/REFimpact/> [Accessed 30 July 2017].

Horowitz, C. R., Robinson, M. and Seifer, S. (2009) 'Community-Based Participatory Research From the Margin to the Mainstream', Circulation. 119: 2633-2642.

A. Ishkanian and S. Szreter, eds. (2012) The Big Society Debate, Cheltenham: Edward Elgar. Johnson, M. T. (2013) Evaluating Culture, London: Palgrave. 
Johnson, M. T. (2017) 'Home', A Cross-Cultural Working Group on "Good Culture” and Precariousness, <http://wp.lancs.ac.uk/good-culture/> [Accessed 30 July 2017].

Johnson, M. T. (2018) "A Cross-Cultural Working Group on "Good Culture" and Precariousness', YouTube, <https://www.youtube.com/channel/UCFYnoCKbEDJAliUeJgBhQ1g/videos> [Accessed 30 July 2017].

Jones, O. (2011) Chavs, London: Verso.

Kelly, G., Mulgan, G. and Muers, S. (2004) Creating Public Value, London: Cabinet Office Strategy Unit.

Kemmis, S., McTaggart, R. and Nixon, R. (2013) The Action Research Planner, London: Springer, pp. 1-32.

Kilburn, D., Nind, M. and Wiles, R. (2014) 'Learning as Researchers and Teachers: The Development of a Pedagogical Culture for Social Science Research Methods?', British Journal of Educational Studies, 62(2): 191-207.

Lewthwaite, S. and Nind, M. (2016) 'Teaching Research Methods in the Social Sciences: Expert Perspectives on Pedagogy and Practice', British Journal of Educational Studies, 64(4): 413-430

Mann, B. L. (2006) 'Case Study Research and Online Learning'. In Case Study Research on Web-Based Learning, ed. B. L. Mann. London: Information Science Publishing, pp. 7079.

McAllister, K. and Vernooy, R. (1999) A guide for monitoring and evaluating participatory research, Ottawa: CBNRM.

McNicoll, P. (1999) 'Issues in Teaching Participatory Action Research', Journal of Social Work Education, 35(1): 51-62.

O’Dowd, S. (2016) 'Communities and the Academy', A Cross-Cultural Working Group on "Good Culture" and Precariousness, 10 ${ }^{\text {th }}$ January, available from: $<$ http://wp.lancs.ac.uk/good-culture/participatory/> [Accessed 30 July 2017].

O’Toole, T. P., Aaron, K. F., Chin, M. H., Horowitz, C. and Tyson, F. (2003) 'Communitybased participatory research', Journal of General Internal Medicine, 18:7, 592-594.

Pain, R., Whitman, G. and Milledge, D. (2011) Participatory Action Research Toolkit, Durham: Durham University Press.

Participatory Methods (2018) 'Glossary', Participatory Methods (Online), Brighton: Institute of Development

Studies,

<http://www.participatorymethods.org/glossary/participatory-action-research>

[Accessed 06 November 2018].

Pearson, N. (2014) 'A Rightful Place', Quarterly Essay, 55: 1-72.

Pobal (2016) 'Area-Based Childhood Programme', Pobal, $9^{\text {th }}$ July, available from: <https://www.pobal.ie/FundingProgrammes/Area\%20Based\%20Childhood\%20\%28A BC\%29\%20Programme/Pages/default.aspx> [Accessed 30 July 2017].

Shore, N., Wong, K. A., Seifer, S. D., Grignon, J. and Gamble, V. N. (2008) 'Introduction to special issue', Journal of Empirical Research On Human Research Ethics: An International Journal 3(2): 1-4.

Silverstein, M., Banks, M., Fish, S. and Bauchner, H. (2008) 'Variability in institutional approaches to ethics review of community-based research conducted in collaboration with unaffiliated organizations', Journal of Empirical Research On Human Research Ethics: An International Journal 3(2): 69-76.

Stake, R. E. 1995. The Art of Case Study Research. London: Sage.

Standing, G. (2011) The Precariat, London: Bloomsbury.

Susman, G. I. and Evered, R. D. (1978) 'An Assessment of the Scientific Merits of Action Research', Administrative Science Quarterly, 23(4): 582-603. 
Tandon, S.D., Phillips, K., Bordeaux, B.C., Bone, L., Brown, P. B., Cagney, K. A., et al. (2007) 'A vision for progress in community health partnerships', Progress in Community Health Partnerships, 1(1): 3-17.

Thames Valley Police (2015) 'Consent: It's simple as tea', YouTube, 16 November, available from: <https://youtu.be/pZwvrxVavnQ> [Accessed 30 July 2017].

Wall, S. (1998) Liberalism, Perfectionism and Restraint, Cambridge: Cambridge University Press.

West, S. E. (2015) 'Evaluation, or Just Data Collection?', The Journal of Environmental Education, 46(1): 41-55.

Yin, R. K. (1984) Case Study Research. Newbury Park: Sage. 